\title{
DEVISING INFORMATION SYSTEMS AND TECHNOLOGY EVOLUTIONARY PATHS WITH IT-CMF
}

\author{
Fábio Longo De Moura and Filipe De Sá-Soares \\ Centro ALGORITMI - University of Minho - Guimarães, Braga, Portugal
}

\begin{abstract}
The importance of Information Systems and Technology (IST) for organizations has been recognized numerous times, whether in facilitating the achievement of strategic goals, or in overcoming operational challenges, in addition to assisting managers in decision-making processes. The sustainable extraction of value from IST requires organizations to make efforts to maintain the use of IST aligned with the organization's objectives. IT-CMF consists of a comprehensive framework to guide the evolution of an organization's exploitation of IST in 36 management areas. Based on this framework, several evolutionary paths for the improvement of the IST capability can be derived. The prioritization and selection among the available paths may pose a problem for organizations, especially due to the intricate relationships between the different areas of intervention. In this study, we resort to Graph Theory to conduct a network analysis of IT-CMF, in order to assist organizations choosing the evolutionary path with the greatest potential for improving their use of IST, taking into account their strengths, weaknesses, and priorities.
\end{abstract}

\section{KEYWORDS}

IT-CMF, Capability, Competency, Graph; Information Systems, Information Systems and Technology

\section{INTRODUCTION}

Improving business performance, or even maintaining the competitive potential, is a daily and constant challenge for an enterprise. To strength its position in the market, an organization needs to change certain characteristics to a different state while preserving those characteristics that meet desirable parameters (Business Dictionary, 2012). This requires managers to devise a path to be followed by the organization towards defined goals. To best support their decisions, managers need information about the current situation and alternative options. Thus, Information Systems and Technology (IST) play a central role in organizations as fundamental resources to the synthesis of information. 
IADIS International Journal on Computer Science and Information Systems

Peppard and Ward (2004) identified three different eras of evolution for organizations, concerning their exploitation of IST, namely Data Processing, Management Information Systems, and Strategic Information Systems. These ages presented distinct challenges for the evolution of organizations, which have been captured in various IST models. Such models depicted scenarios that might be faced by organizations and suggested paths for their evolution in the use of IST, with the expectation of achieving success in different aspects of the organization's operating environment. As complexity increased inside and outside organizations, and the amount of available information surged, organizations experienced a greater dependence on IST, which imposed a stronger need for better management of their information-related resources.

To help organizations respond to this information management challenge, new evolution models and maturity models that formulate relationships between IST and organizations emerged. One of the latest models is the Information Technology Capability Maturity Framework (IT-CMF). The design of the IT-CMF reflects the demand for differentiated analyzes about the possibilities of improving organizations by the exploitation of IST. The framework indicates knowledge and skills that an organization should hold in order to manage efficiently and effectively the organization's IST function (Duarte and Da Silva, 2013). According to the provisions of the IT-CMF (IVI, 2016), it is possible to carry out diagnostic and in-depth analysis for each of the 36 management areas that make up the framework. The framework also points out relationships between management areas and how improvements in one area may contribute to improvements in other areas. The organization that wishes to evolve, based on new demands or on some factor that influences the alteration of any of its characteristics, should consider evolution paths that may enhance certain management areas that make up the IT-CMF, with the prospect of rising its business performance. However, there may be alternative paths to choose from, taking into account not only the objectives established for the areas of IT-CMF, but also considering the relationship between the areas. Given the interdependences between the 36 management areas of IT-CMF, organizations may face a complex task identifying and making sense of different evolution paths. From this observation stemmed the motivation to carry out this study. Faced with this challenge, the research question that this paper aims to answer is: What is the best evolutionary path that an organization may follow to improve its IST capability, in the context of using the IT-CMF?

To facilitate the selection of evolutionary paths by organizations through better use of IST, we conducted a network analysis of IT-CMF, resorting to Graph Theory in order to make explicit the interrelationships, scope, and possibilities when prioritizing improvements in one or more areas of IST in an organization. The combination of IT-CMF with Graph Theory also allows managers more clarity in decision making, in addition to greater assertiveness and efficiency in the deployment of resources for the evolution of organizations through IST.

The following section covers the literature review, setting the stage for a new way to apply IT-CMF according to the specificities of each organization. After the literature review, this work is composed of two other sections, related to Graph Theory and to the proposition of using Graph Theory to perform a network analysis of IT-CMF, with the aim of improving the application of IST organizational resources and highlight different views about the relevance of each area of IT-CMF. At the end of the paper, conclusions are drawn and future work involving the application of this proposal is advanced. 


\section{LITERATURE REVIEW}

In this section, organizational success and its measurement are discussed, taking into account the role that IST play both for that end and in that task. Then, evolution and maturity models for the exploitation and management of organization's IST are briefly reviewed. Afterwards, the structure of IT-CMF and the relationship between the areas composing this framework are put into perspective.

\subsection{Organizational Success}

The business practices of organizations must keep up with constant changes, as practices that are known to be successful in the past may not indicate success in the future (Fong, 2010), as illustrated by what happened to companies with predominant market share in the recent past, such as Kodak, Blockbuster, and BlackBerry. Previously, Kodak had an $80 \%$ market share for camera film, coupled with $85 \%$ of cameras sold on the market. Blockbuster, once one of the best-known brands in the United States of America, decided to maintain the business model that led it to success, ending up declaring bankruptcy after 25 years of existence. Besides not being able to keep up with the transformation that was taking place in its environment, Blockbuster chose to maintain the organizational parameters and decisions that have led to success in the past, but which proved to be inadequate in the face of competition. On the other hand, BlackBerry, which between 2000 and 2010 dominated the mobile device market, being recognized for the constant innovation in its products, was unable to keep its operating system competitive in the face of new competitors iOS and Android. In 2017 it held only $0.01 \%$ of market share, and today, despite still manufacturing and selling smartphones, its core business is in the field of security and Internet of Things (IoT).

The measurement of the organization's success should not be static and should not be based on simplistic and isolated measures. A tool widely used to measure the success of organizations is the Balanced Scorecard (BSC) (Kaplan and Norton, 1992). BSC aims to overcome weaknesses of indicators such as Return over Investment (ROI) and Earning per Share (EPS), noting that these are not sufficient indicators for continuous improvement and innovation. The BSC authors advocate the integration of complementary indicators to assist in the evaluation of the success of the organization, taking into account the:

- Customer perspective: It refers to translating the company's mission into measures that directly affect the customer, such as time, performance and service, quality, and cost;

- Internal business processes perspective: It refers to meeting customer expectations, resulting in their satisfaction. Some factors are related to the identification of the company's competencies and the technologies in use;

- Learning and growth perspective: It involves identifying the main parameters that demonstrate competitive success. It must be constantly updated to force the continuous improvement of the organization's products and processes;

- Financial perspective: Regarding financial goals (profitability, growth, and shareholder value), the organization's strategy is evaluated to prove its effectiveness.

When composing a set of indicators of the organization's success, it makes sense to consider the external environment, in order to compare the organization with its the competitors (Neely, Gregory and Platts, 2005). One way to consider the external environment is to add the following indicator: 
IADIS International Journal on Computer Science and Information Systems

- Competitor perspective: What are our competitors doing? The need to follow what competitors are doing had already been pointed out by (Hamel and Prahalad, 1994), noting that the organization's survival is conditioned on identifying the needs of customers in the future, so to create new opportunities and dominate emerging ones.

Considering another organizational success approach, proposed by Sink (1985), there are different perspectives to measure the success of the organization, which include analysis of product quality, customer satisfaction, capacity for innovation, quality of work, employee retention, the perceived value of goods and services, operational efficiency and social responsibility.

The success of an organization depends on its sustainability and growth, requiring the orchestration of very different tasks, such as segment identification and market niche, development of products and services, acquisition of resources, development of operating systems, development of management systems, and development of corporate culture (Flamholtz and Aksehirli, 2000). Flamholtz and Aksehirli suggest a set of tasks that must be done in successful organizations, divided into six levels, represented in a pyramid, as shown in Figure 1. The base of the pyramid encompasses the essentials for successful organizations, while the highest levels contain attributes that form the organization's infrastructure, responsible for limiting the possibility of imitation by competitors.

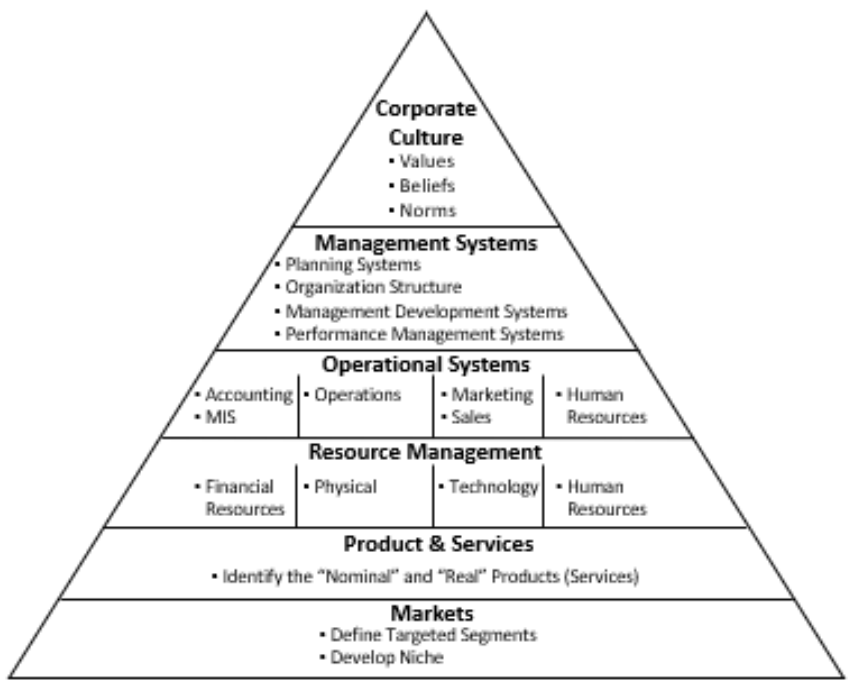

Figure 1. Pyramid of Organizational Development: The Six Key Building Blocks of Successful Organizations (Flamholtz and Aksehirli, 2000)

Alongside what was argued by Flamholtz and Aksehirli (2000), we may also consider the theory proposed by Barney (1991), stating that the organization's resources and capabilities are what will make it possible to differentiate itself from competitors. Barney also mentions the possibility of organizations being heterogeneous concerning their resources. This proposal was named Resource-Based Theory (RBT), to which other authors later used in their studies. Peppard and Ward (2004), for example, note that RBT considers the accumulation and implantation of resources that match the idiosyncratic (unique) resources of proprietary assets and provide the source of sustainable competitive advantage. This perspective contrasts with the levels of the organizational development pyramid proposed by Flamholtz and Aksehirli 
(2000), specifically at the higher levels of the pyramid, which must be prioritized after the organization has already performed the tasks forming the lower levels of the pyramid.

As input to the measurement of the level of success, which in itself has become more demanding since it has to consider a multitude of parameters (Curley, 2008), as instruments to know what is happening, or as products or services by themselves, IST play a fundamental role in the success of organizations. It is not surprising, therefore, the emergence of models to guide the exploitation and management of organizations' IST.

\subsection{Evolution and Maturity Models}

The observation of changes in the characteristics of organizations, which occurred over several decades, led to the adoption of the concepts of evolution and maturity in order to envision the progress of an organization and its sophistication regarding the use of IST. Initially approached by Nolan (Nolan, 1973, 1979; Gibson and Nolan, 1974), the concept of evolution was present in several models that aimed to guide the progress of organizations in using IST.

Evolution, applied to the organizational context, refers to the process of change that the organization experiences over time, involving the alteration of one or more characteristics, and that may be marked by false starts and present different directions, while hoping to maintain what is believed to be working (Business Dictionary, 2012).

Later, the concept of maturity was used, replacing the concept of evolution, as a means to structure the models describing the progression in the use of IST. This change reflects a new approach adopted in the models in what concerns the way to evaluate the organization. While models of evolution only classify the organization in a stage, maturity models emphasize the measurement of the quality of the relationship between the organization and IST, contemplating factors that directly or indirectly influence this relationship, and suggesting actions for its improvement, in order to raise the evolutionary stage of the organization. The factors that help to assess the evolutionary stage of organizations in what concerns IST, are usually called variables, and guide organizations in answering the following questions: Where are we?, Where are we going?, and What will we do to get there? (Santos and Valdesuso, 1985).

Technological developments place continued pressure on organizations, forcing them to question their current and future use of IST. These developments lead to the accelerated obsolescence of models designed to support the evolutionary or maturing process of organizations regarding their use of IST, as factors that were previously relevant may no longer make sense. The plethora of possibilities for the progress of organizations makes the proposal of directions that unveil evolutionary paths a challenging endeavour. Indeed, the scope of most of the models designed so far proved to be limited due to the numerous possibilities provided by the use of IST. Ergo, there is a need for a holistic approach to extract value from IST, that pays attention to different sectors of the organization, their specific demands, and relationships, making explicit the influences and dependencies existing between them.

\subsection{IT-CMF}

Based on various scientific contributions produced over decades of studies on the relationship between organizations and IST, the IT-CMF appears as a composite of maturity models, not limited to analyzing a specific context. The framework architecture includes 36 contexts for analysis, each with a maturity model that indicates a possible path to be taken by the 
IADIS International Journal on Computer Science and Information Systems

organization that seeks to improve its relationship with IST in a management area (Carcary, Doherty and Thornley, 2015). Each IT-CMF maturity model is composed of five evolutionary stages, and the areas covered by the framework have guiding questions to assess the level of maturity, guidelines for the evolution of the area, typical challenges for the intended evolution, as well as actions necessary to be performed by those responsible for the evolution of the organization. The areas, called Critical Capabilities (CC), are organized into four large groups, called Macro Capabilities (MC). These groups bring together areas that have equivalent objectives, which according to the provisions of IVI (2016), are:

- Managing IT like a business (MC1): Composed of $14 \mathrm{CCs}$, it proposes to change the vision of how IT is considered, migrating from a cost center to a value center view, offering solutions to customers and existing problems in the business;

- $\quad$ Managing the IT budget (MC2): Formed by 4 CCs, it includes challenges related to the cost of IT, such as unplanned costs, maintenance of legacy systems, and the difficulty of strategically investing in new technologies;

- Managing the IT capability (MC3): It covers 15 CCs and refers to the maintenance of the services provided by the IT function and the development of new services, stimulating innovation in the organizational environment through services and solutions provided by IT; and

- Managing IT for business value (MC4): It involves 3 CCs, which aim to ensure that IT investments will enable business benefits, fostering the view that IT projects are not technology projects, but projects that generate business value.

The process of diagnosing the necessary improvements for the organization, which is also included in the framework architecture, will indicate a set of CCs that should be improved, according to the current situation of the organization and its business objectives. The advantages for the Chief Information Officer (CIO), through diagnosis and improvements in the organization using the IT-CMF, are a focus on business value, instead of cost; sustainable IT; an emphasis placed not on value generated by cost, but on optimized value, and a view of IT as an organization's core competence.

When using the IT-CMF, it is possible to analyse the organization in two different ways, namely, diagnostic analysis and in-depth analysis, with the latter adding to the diagnosis the definition of an action plan to improve the critical capabilities for the analysed organization. Not being mandatory, the IT-CMF can serve as a guiding compass for managers in the daily challenges of the organization (Curley and Kenneally, 2011). It is stressed, through IT-CMF, the possibility of the organization being experiencing an evolutionary process, which may contain characteristics of two levels of maturity.

The analysis of the relationships between the CCs reveals how different CCs may contribute for the improvement of the organizational use of IST. Many of the contributions may come from isolated actions, however, they may have comprehensive results. The relationships between the 36 CCs that make up the IT-CMF involve 230 contributions, either between CCs from the same MC or between CCs from different MCs, as shown in Table 1. Regarding the relationships between the CCs and the conduction of the organization's in-depth diagnosis, it becomes possible to prioritize improvements of specific CCs, focusing on the development and implementation of improvements in the systemic relationships that emerge from the various CCs. The IT-CMF is, therefore, a practical, action-oriented tool, directing the organization towards business innovation and differentiation through IST as part of a process of maximizing the CCs, to expand the organization; s business, with IST being a strategic resource and support in the process of innovation and change (Carcary, Doherty and Thornley, 2015). Considering 
the significant number of relationships between the CCs, the prioritization of CCs on the path to be followed for improving areas of the organization becomes a complex task. Therefore, it is necessary to understand better such relationships, specifically regarding the contributions provided and received by each $\mathrm{CC}$, in order to assist managers in the selection of the best evolutionary path to follow. This argument is related to the purpose of IT-CMF - to do more with less - making it possible to add value to the business through an assertive investment in IST.

According to the data provided in Table 1, the relevance of MC1 is patent, both in the contributions provided by the CCs that compose it and in the contributions received by their CCs. On the other hand, there is a lower weight of the CCs of $\mathrm{MC} 2$, regarding the contribution to the other CCs, as well as the contributions received by the CCs that make up MC4. To enable a better representation of the CCs, visually indicating the relationships between them and making clear their contribution to improvement actions, we resort to Graph Theory, using representations as well as applying algorithms on the data compiled from IT-CMF. The application of algorithms enables the identification of communities of CCs that show greater proximity, the recognition of CCs with greater relevance in the framework, either in general or in the contributions received or provided, as well as unveiling the affected CCs through the improvements made in a single CC.

We argue that Graph Theory applied to the IT-CMF will assist in the provision of directions for the evolution of the organization, putting into perspective the strengths and weaknesses of the organization to its strategic goals. The planning of actions to be performed can also be supported by the representations elaborated using the concept of graphs, namely by defining the evolutionary path to be followed, according to the CCs involved. The construction of the graph representations that will appear later in the paper were made using the application Gephi (version 0.9.2).

Table 1. Contributions between IT-CMF MCs

\begin{tabular}{lcccc}
\hline $\begin{array}{l}\text { Contributions } \\
\rightarrow\end{array}$ & MC1 & MC2 & MC3 & MC4 \\
\hline MC1 & 52 & 8 & 47 & 7 \\
MC2 & 6 & 6 & 2 & 3 \\
MC3 & 34 & 2 & 45 & 1 \\
MC4 & 4 & 7 & 4 & 2 \\
\hline
\end{tabular}

\section{GRAPH THEORY}

Graph Theory is a branch of mathematics concerned with the study of structures - graphs - that model relationships between objects. A graph is a representation formed by a set of points (vertices) and connections between points (edges), which can be used to represent different situations in the real world (Bondy and Murty, 1976). The history of Graph Theory began in 1736, in the famous dilemma of the Königsberg bridges. In this dilemma, the seven bridges (edges) and four regions (nodes) of the city of Königsberg, cut by the river Pregel, were involved (Goldbarg and Goldbarg, 2012). Figure 2(A), developed by Rouse Ball (Gross and Yellen, 2003), exemplifies the dilemma mentioned when he called the concept of graph. This example came from the challenge of the Königsberg population, which was to walk through all regions of the city without crossing the same bridge more than once. 
One of the most used examples for graphs is about representing people, with lines modeling the relationship between people, thus being able to demonstrate large concentrations and displacements on a map. Such a scenario is of great relevance for macro analysis, such as those related to the spread of diseases in the world. A current case of application is the evolution of the pandemic caused by the SARS-CoV-2 virus (COVID-19). When traveling, people end up transporting the virus to their localities, but passing by large points of concentration of people, such as large airports (hubs).

Besides being heavily used for modeling and analyzing maps, another example of the application of Graph Theory is to chess games, with each piece having a specific graph that indicates its displacement. The Queen, for instance, is the piece that has the greatest number of opportunities in its displacement on the board and may have up to eight possible edges and several vertices for its final position on the board, as shown in Figure 2(B). The queen is at the center of the graph, indicated by the filled black dot, having 25 vertices as potential final positions, depending on whether there is an impediment to displacement, caused by any other piece of the board.

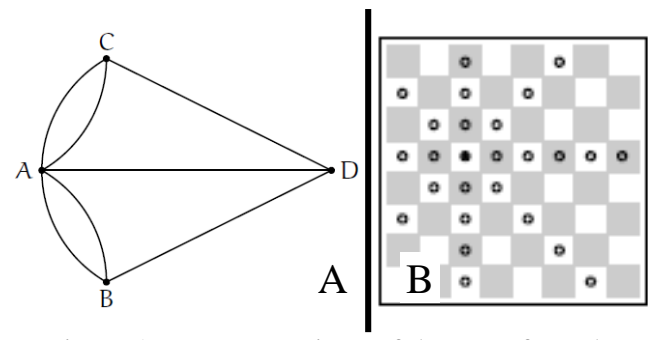

Figure 2. Representations of the use of graphs

These are just a few examples of the use of graphs, from a myriad of applications in chemistry, operations research, social sciences, social media, and computer science, to name a few. In this work, graphs will be used to represent the CCs and MCs of IT-CMF, to clarify how $\mathrm{CCs}$ are related and how they can indicate paths for the evolution of organizations, in what concerns IST. The path to follow is based on the diagnosis of the improvements to be made, according to the objectives defined for the organization. In other words, the graph makes clear a sequence of actions related to the various CCs that make up the IT-CMF, considering the improvements identified as necessary by the organization. It may also indicate required routes that pave the way for the evolutionary path sought by the organization, that is, in order to follow the selected path, it might prove essential to mobilize beforehand certain resources that improve specific areas of the organization on which that path is dependent.

\section{A NETWORK ANALYSIS OF IT-CMF}

The use of graphs to assist in the study of various areas of knowledge, as already mentioned, is not new. In this work, given the multiple alternative paths available to the organization to improve its exploitation of IST, the use of Graph Theory proved a viable means to address two issues. The first was the identification of alternative evolutionary paths that an organization may consider. The second was the definition of a path that prioritizes the improvements to make in the organization, considering the relationships between the areas of the IT-CMF as shown in Table 1. 


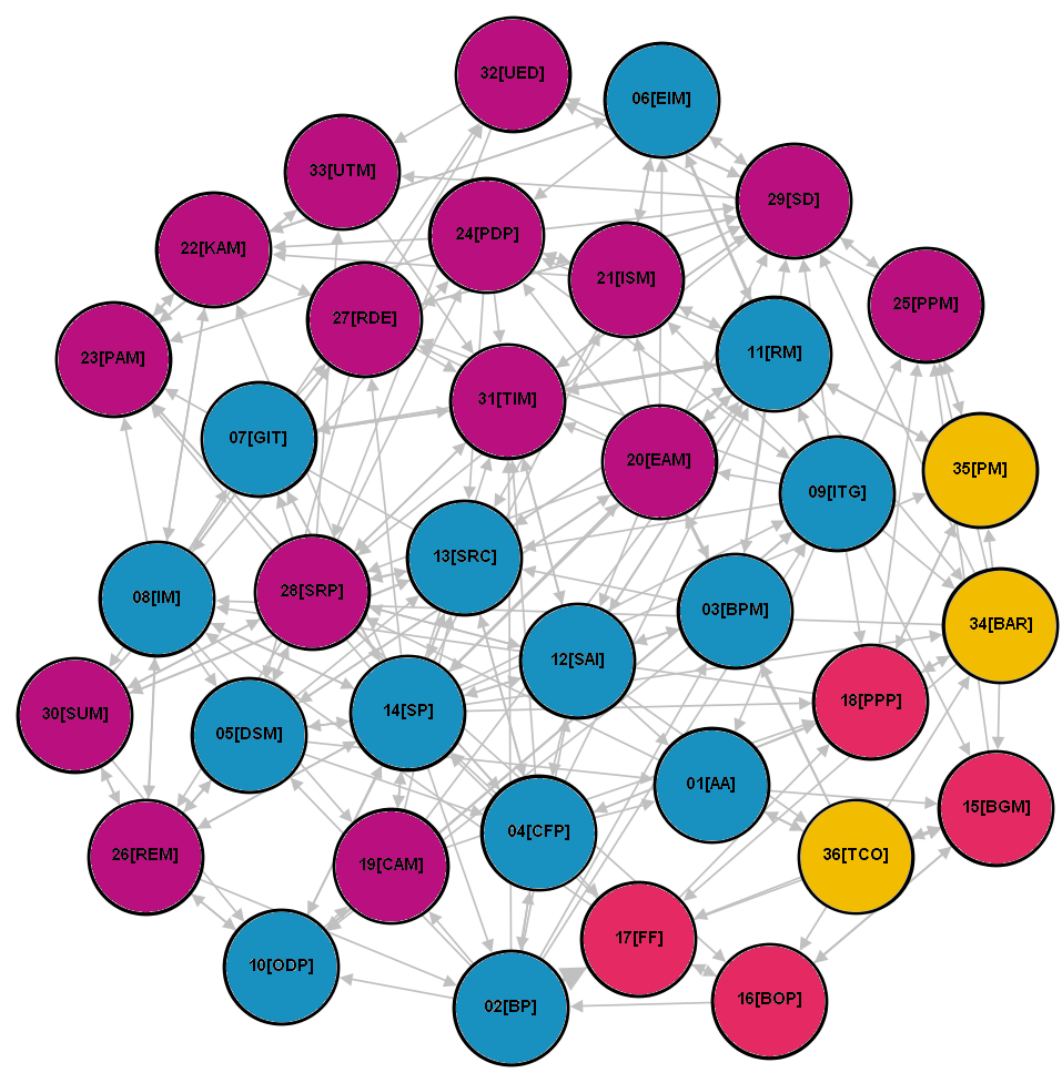

Figure 3. IT-CMF Critical Capabilities

Figure 3 represents the IT-CMF, without applying any Gephi resources to differentiate the CCs, except using specific coloration for the CCs, according to the MCs to which they belong. The only resource applied refers to the contributions provided between the CCs, represented by the arrows. According to the direction indicated by the arrows $(\leftarrow$ or $\rightarrow$ ), the picture depicts how the contribution from one $\mathrm{CC}$ to another $\mathrm{CC}$ occurs. Whenever the arrow points to both sides $(\leftrightarrow)$, it means that there is a mutual contribution between the involved CCs.

Hence, it became possible to analyze all the CCs that make up the framework, as depicted in Figure 3, as well as a subset of CCs, such as those that make up each MC, as shown in Figure 4. In the figures, $\mathrm{CCs}$ are identified by a numeral and an abbreviation in square brackets (the meaning of each abbreviation is provided in Appendix A).

In both Figures 3 and 4, CCs are arranged following the distribution proposed by the Force Atlas 2 algorithm. In Figure 4, the $\mathrm{CCs}$ are dimensioned according to their degree (corresponding to the inputs and outputs of each node $-\mathrm{CC}$ ). The color of each node corresponds to the application of the Heat Map, with an emphasis on the $\mathrm{CC}$ with the highest degree in the analysis performed, except for MC2. This exception results from CCs BOP(16) and $\mathrm{FF}(17)$ having the same degree, so they are dimensioned and colored based on their centrality.

The analysis of Figure 4 suggests that the CC SP (14) has great relevance in the MC to which it belongs, and this relevance is reinforced when considering Figure 5, which, like Figure 4, 
IADIS International Journal on Computer Science and Information Systems

scales the CCs according to their grade (input and output). It is interesting to note the difference in the relevance of some CCs, concerning their contribution to their MC and to the entire IT-CMF. When looking at MC1 (Figure 4), for example, the CCs EIM (06) and IM (08) have low relevance, while for the framework (Figure 5) their relevance is visibly higher.

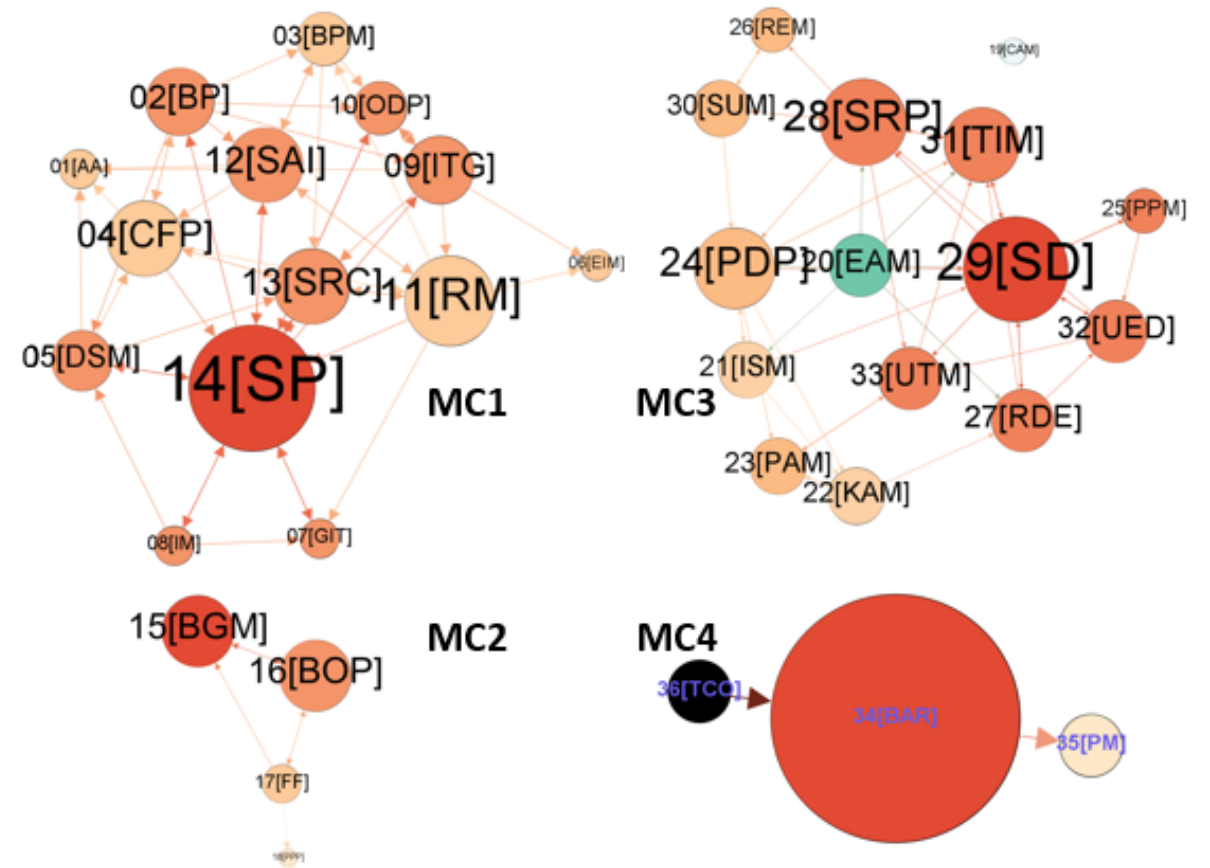

Figure 4. IT-CMF MCs and their CCs

Another behavior that deserves to be highlighted is the relevance of the CCs that make up the MC2. When analysed together with the other CCs in the framework, the inversion of the relevance of the CCs is observed when considering their degree (input and output) in the graph. The CC PPP (18) is the least relevant in the MC that belongs to, however, compared to the other MC2 CCs (cf. Figure 5), it is the MC2 CC with the greatest relevance, followed by the CC FF (17), the second with less relevance in MC2. Regarding the CCs that make up the MC3, the relevance of each one, according to their input and output degree, both for the MC and to the entire framework, is equivalent. The graphs also reveal that CC SP(14) has great relevance in the framework, as well as for the MC in which it is located. It is noteworthy that CCs $\operatorname{SRP}(28)$ and $\mathrm{SD}(29)$ also reflect an equivalent degree of importance for the MC, but in the global framework they are less important. In this case, considering the need to improve CCs that belong to MC2 and MC4, perhaps the best path does not involve these CCs, since it offers only one contribution to MC2 and receives only one contribution from MC4.

Concerning the contributions between CCs, CC CAM(19) does not co-participate in the MC in which it is located, exercising relations in large part with the CCs of MC1 (seven CCs) and MC3 (one CC). This fact strengthens and clarifies the essence of the framework, namely that the relationships between different sectors enable the organization to evolve. Thus, although CC $\mathrm{CAM}(19)$ works to raise the quality of MC3, its improvement will also facilitate improvements intended for MC1 and MC3. 


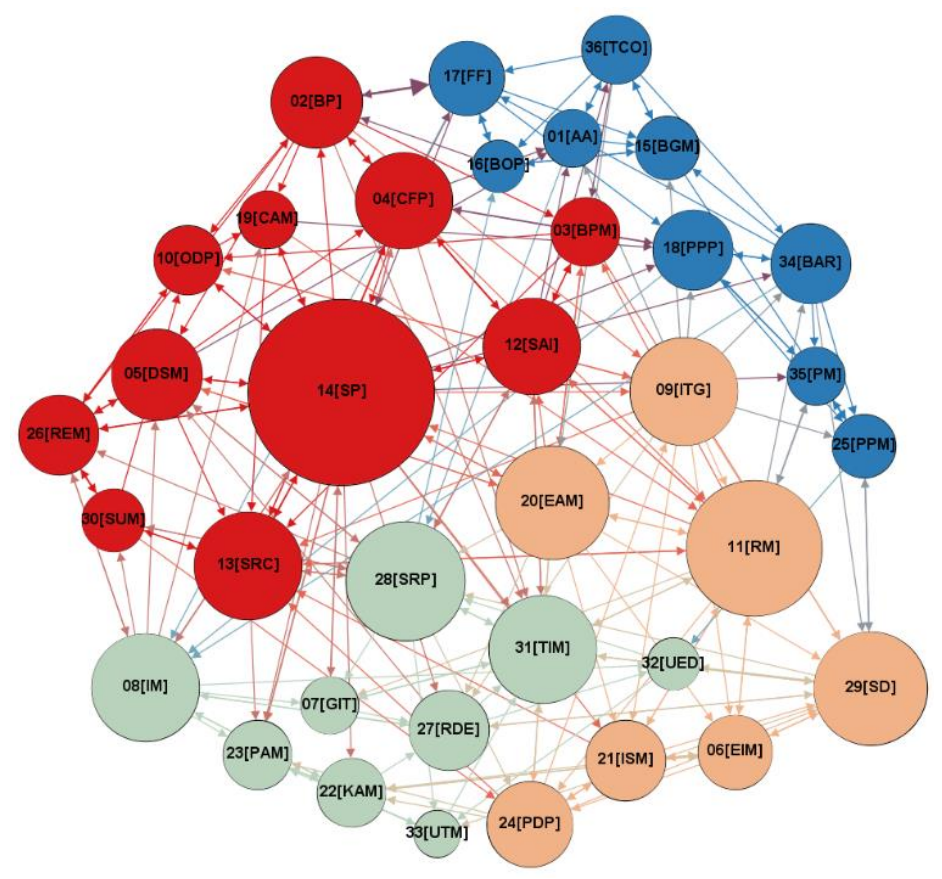

Figure 5. IT-CMF CC Communities

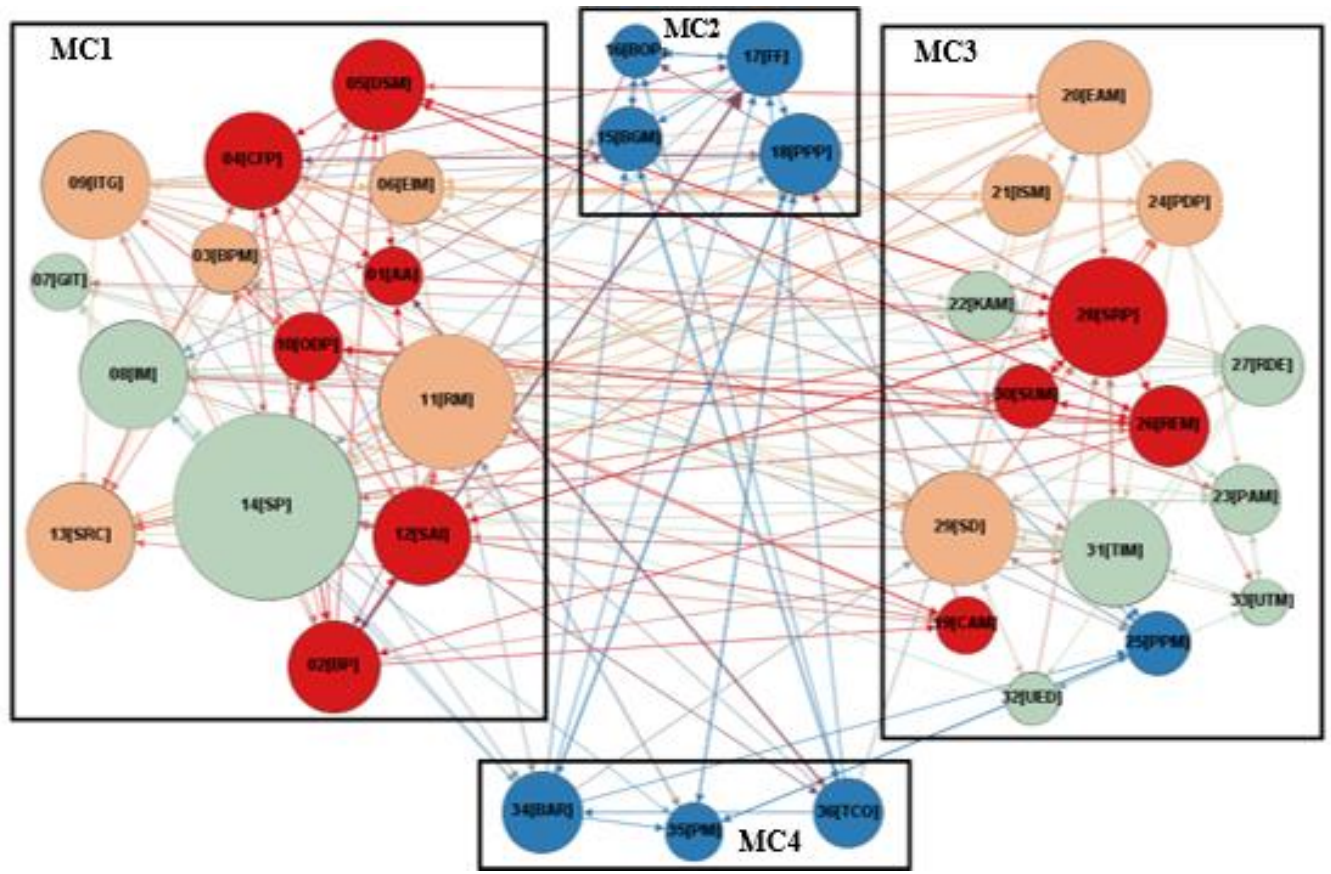

Figure 6. Organization of CCs Communities according to MCs 
IADIS International Journal on Computer Science and Information Systems

Figure 5 stresses this statement, presenting another point of view on the IT-CMF CCs. In the Figure, the CCs are presented in colors that represent their communities, rendering the CCs that have the strongest relationship. This result was obtained through the distribution using Force Atlas 2 algorithm and the calculation of Modularity (0.238). It is noted, therefore, the existence of different communities from the corresponding organization with the MCs (presented in Figure 6), emphasizing again the argument that although each $\mathrm{CC}$ has a main objective, justifying its classification in an MC, its evolution may contribute to the improvement of other area of the organization. An additional finding emanating from Figure 6 is that all the CCs of MC2 and MC4 pertain to the same community. This highlights the mutual collaborations that occur between the CCs of these two MCs.

Still regarding the communities that were identified, as a consequence of the strong relationship between $\mathrm{MC} 2$ and $\mathrm{MC} 4$, there is also a strong relationship between the CCs of MC1 and $\mathrm{MC} 3$, which are the ones with the highest number of CCs in the framework, respectively 14 and 15 CCs. However, there are groups of CCs with greater connectivity, which led to the identification of three other communities of CCs in the graph.

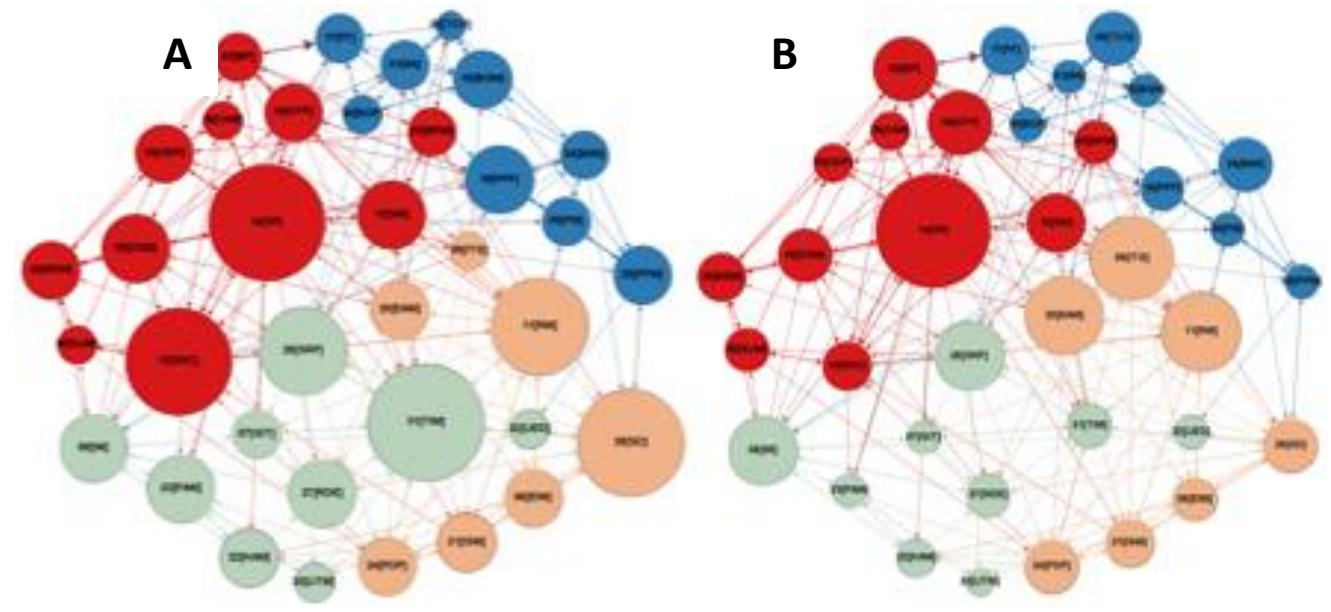

Figure 7. Input and Output Degree Comparison of CCs

Another representation derived from Figure 5 and shown in Figure 7 is the scaling of the nodes according to their degree, dividing it into two figures, one to represent the degree of inputs (A) and the other to represent the degree of outputs (B). This representation assists to the definition of paths to be followed to carry out activities related to the CCs, considering what should be prioritized in an evolutionary path of the organization. For the sake of illustration, consider the following scenario: let us imagine that managers identify as necessary to make improvements in $\mathrm{CC} \mathrm{BPM}(03)$, belonging to $\mathrm{MC} 1$. Knowing that BPM is part of a community (resulting from the modularity of the graph) whose main facilitators are $\mathrm{CCs} \mathrm{BP}(02), \mathrm{CFP}(04)$, SAI(12), SRC(13), and SP(14), it makes sense to consider and evaluate the contributions provided by these CCs, which will help to improve the organization's BPM(03). For example, $\mathrm{CC} \mathrm{BP}(02)$ contributes directly to $\mathrm{CC} \mathrm{BPM}(03)$ and can assist in its evolution. This possibility of an evolutionary path is represented in Figure 8(A). On the other hand, the contribution provided by $\mathrm{BPM}(03)$ to the other $\mathrm{CCs}$ should also be considered, regarding the relevance of these CCs to the organization. In this case, contributions resulting from improvements made in 
the context of $\operatorname{BPM}(03)$ are directed to $\operatorname{CCs} \operatorname{ODP}(10), \operatorname{SAI}(12), \operatorname{SRC}(13), \operatorname{EAM}(20)$, and $\mathrm{SD}(29)$. If these CCs are also considered relevant to the business, the importance of using resources to improve $\mathrm{BPM}(03)$ is even greater. This path possibility is shown in Figure 8(B).
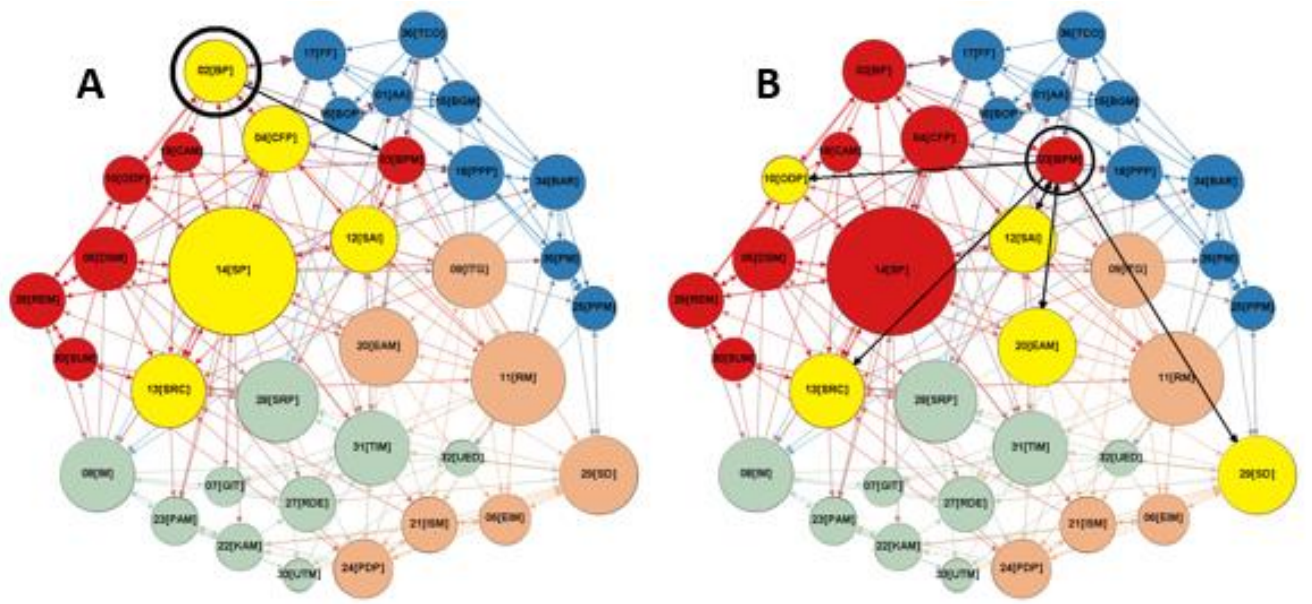

Figure 8. Improvement Proposals involving BPM(03)
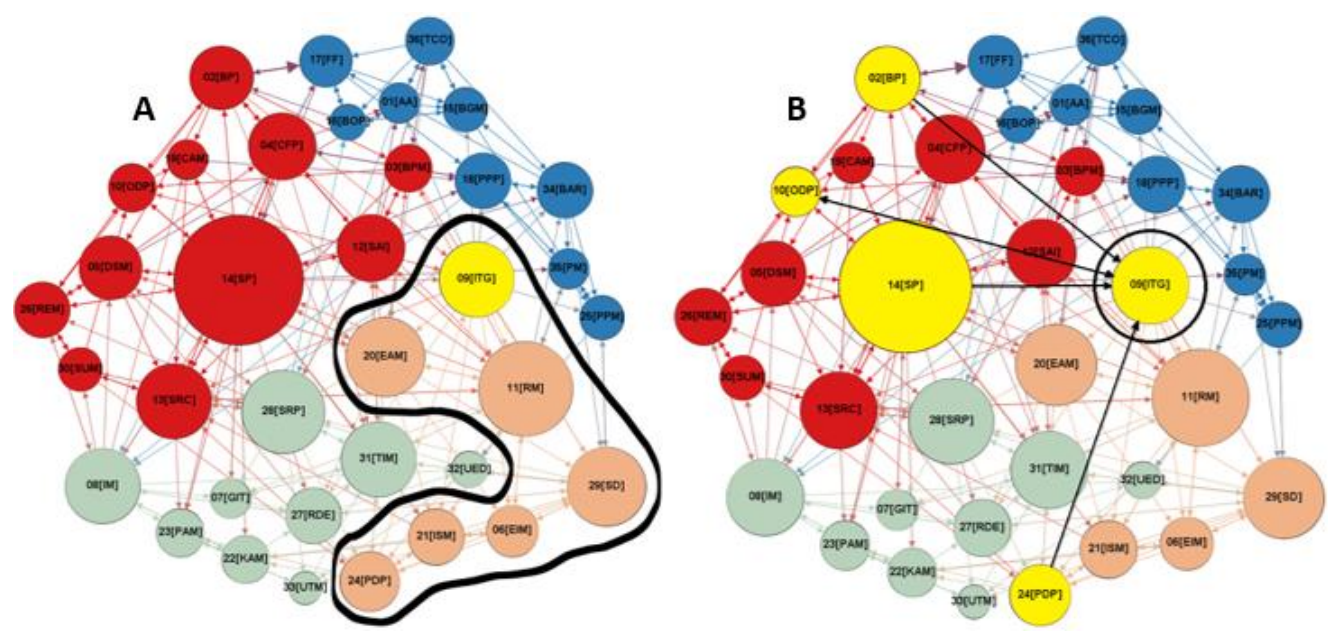

Figure 9. Improvement Proposals involving ITG(09)

Another important insight is that $\mathrm{CC} \operatorname{EAM}(20)$ has a high degree of output, contributing to twelve other CCs, as shown in Figure 7(B). In another scenario, considering the necessity to make improvements in some of the areas that make up the IT-CMF, the need for improvement in the CC ITG(09) is adopted as an example. One of the possibilities is to consider the community in which this CC is located, and, therefore, work to improve other six CCs, namely: $\operatorname{EIM}(06), \operatorname{RM}(11), \operatorname{EAM}(20), \operatorname{ISM}(21), \operatorname{PDP}(24)$, and $\operatorname{SD}(29)$. In this proposal, which is represented in Figure 9(A), there would be the strengthening of the desired CC (ITG) through the proximity of the areas worked. Another possibility, represented in Figure 9(B), is to identify 
IADIS International Journal on Computer Science and Information Systems

which CCs are suppliers of improvements for the $\mathrm{CC}$ in question, which in this case are four CCs: BP(02), ODP(10), SP(14), and PDP(24). The decision on the way forward may be based on the objectives of the organization or on the forecast and availability of resources to improve the CC ITG(09). An assessment of the level of maturity of the organization in the sets of CCs can be an important factor to assist in the decision, since the strengths and weaknesses of the organization in different areas can be identified, facilitating the achievement of improvements in the desired area. By choosing the path that involves the stronger points, in terms of IT-CMF $\mathrm{CCs}$, it is possible to establish synergies at an early stage, involving the CCs with greater strength in the organization. Later, the organizational efforts may expand improvements to other areas, leveraging the results of those synergies, either by harnessing the strengths diagnosed or by the improvements made in a group of CCs.

Concerning the relevance of MCs to the framework, it is evident that MC1 and MC3, by efficiently and effectively employing organizational resources in the evolution of their CCs, tend to enable a radical change in the organization. However, although MC2 and MC4 have substantially fewer CCs, when employing organizational resources to improve these CCs, the contributions to the other CCs in the framework are above the average of the number of contributions provided by the $\mathrm{CCs}$ of $\mathrm{MC} 1$ and $\mathrm{MC}$. Table 2, as a complement to Table 1, shows the average of contributions per CC, taking into account the number of CCs in each MC.

Table 2. Average contributions from CCs (Grouped by MCs)

\begin{tabular}{lcccc}
\hline $\begin{array}{l}\text { Average } \\
\text { Contributions }\end{array}$ & MC1 & MC2 & MC3 & MC4 \\
$\rightarrow$ & & & & \\
\hline MC1 & 3.71 & 0.57 & 3.36 & 0.50 \\
MC2 & 1.50 & 1.50 & 0.50 & 0.75 \\
MC3 & 2.27 & 0.13 & 3.00 & 0.07 \\
MC4 & 1.33 & 2.33 & 1.33 & 0.67 \\
\hline
\end{tabular}

Considering the contributions featured in Table 2, one notes that the relationship between the CCs of MC1 and MC3 are very strong, as well as the relationships that exist internally, that is, between the CCs of MC1 and MC3 themselves. However, when directing the relationship between these MCs and the other smaller MCs, the contribution is much lower than the contributions provided by the MCs composed of a lower number of CCs. The weakness, nevertheless, is part of the internal relations between the CCs, since, comparatively, the smaller MCs have, on average, fewer internal relations than the most numerous MCs. Therefore, when defining an IST improvement plan in an organization, one must bear in mind the contributions provided by the smaller MCs, since these MCs may have a more comprehensive impact on the potential results, once organizational resources are properly applied. Indeed, considering the CCs with the highest degree in the graph, in MC2 and MC4, respectively PPP(18) and BAR(34) (see Figure 4), and also because they are in the same community (see Figure 5), it is advisable that, even if such CCs are not prioritized, the contribution provided by them needs to be taken into consideration, as it is possible that the objective to be achieved can be facilitated when attention is paid to these CCs.

To clarify the relationships and contributions existing between the CCs of the MCs that make up the IT-CMF, Figure 10 makes a comparison of the direct relationship between the MCs. In Figure 10(A) the thickness of the edges (the size of the edge arrow) conveys, through the direction of the arrow, the contribution provided from one MC to another. It can be noted, 
therefore, the strength of the relationship between $\mathrm{MC} 1$ and $\mathrm{MC} 3$, as previously mentioned, in addition to also demonstrating the connectivity of the CCs that make up MC1 and MC3 (as revealed by the self-loops). Figure 10(B) depicts the total contributions divided by the number of CCs that make up the MC. In this representation, it is evident that, in all relationships, MC2 and $\mathrm{MC} 4$ provide more contributions to $\mathrm{MC} 1$ and $\mathrm{MC} 3$ than receive contributions from these MCs, making clear the need to provide attention to MC2 - Managing the IT Budget and MC4 - Managing IT for Business Value, even though, quantitatively, they are less expressive than the other MCs. The CCs that make up MC2 and MC4 can act as great facilitators for improvements to be performed in different areas of management of the organization.

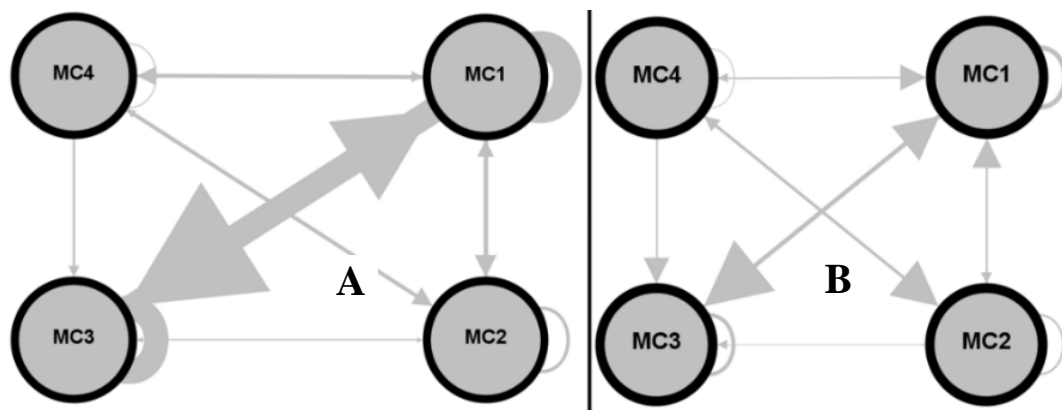

Figure 10. Relations and Contributions between MCs and CCs

\section{CONCLUSION}

In the face of a challenge, it is necessary to act. To act, the action must be well-founded, that is, be based on elements that support that the action performed was identified as the best option for the given moment. The decision for a path to be followed to make improvements in the organization needs to be as assertive as possible, as many organizations have no margin for errors, or, even if errors are part of the evolutionary path that the organization takes, they are never desirable. The proposal to use graphs as an aid in finding the evolutionary paths for the organization, which are adapted to its needs, seeks to maximize the use of organizational resources in the adoption and use of IT-CMF.

Considering that IT-CMF is one of the most robust and mature references in the evaluation of the relationship between organizations and IST, it is of great relevance to explore it, and to present different points of view for its application, seeking to facilitate the immersion of managers in the content provided. By conducting a network analysis of IT-CMF based on Graph Theory, it is possible to demonstrate, with greater clarity, how the management areas that make up IT-CMF are related, as well as revealing the paths that can be covered by the organization, to improve its use of IST. The possibilities for combining the graph concept with the framework are numerous, and it is believed that sufficient guidelines have been provided in this paper to suggest how managers can build the best roadmap to be followed, deploying organizational resources as efficiently and effectively as possible, focusing on the creation of aggregate value to the business by using IST. To this end, it is essential to identify the organization's strengths 
and weaknesses, relating them to strategic objectives, so that the application of resources in the desired improvement is as efficient as possible.

The evolutionary path to be defined involves several planned outcomes, in addition to the use of resources and actions by the managers. Although there are numerous possible evolutionary paths suggested by the application of Graph Theory to IT-CMF, managers are required to consider and detail such outcomes, resources, and actions foreseen for the evolutionary path, taking into account the practices suggested by IT-CMF. This will help to reduce uncertainty and eventual surprises resulting from superficial analyzes of the organization.

For future work, we consider the application of this proposal in different organizations to be extremely relevant. This would assist in the validation of the proposal of using Graph Theory for planning improvement actions to be carried out based on the IT-CMF. We also consider valuable to involve professionals to classify the importance of contributions between the CCs, resulting from the improvement actions in each one. The result of this classification will help to define weights for the edges of the graphs representing IT-CMF. If such a weighted graph is available, the definition of an evolutionary path should result facilitated, perhaps being feasible the application of graph shortest path algorithms that may take advantage of those weights to find the most sustainable and fruitful improvement actions in CCs.

\section{ACKNOWLEDGEMENT}

This work has been supported by FCT - Fundação para a Ciência e Tecnologia within R\&D Units Project Scope: UIDB/00319/2020.

\section{REFERENCES}

Barney, J., 1991. 'Firm Resources and Sustained Competitive Advantage', Journal of Management, Vol. 17, No. 3, pp. 99-120.

Bondy, J. A. and Murty, U. S. R., 1976. Graph Theory with Applications. Ontario: Macmillan.

Business Dictionary, 2012. Evolution. Available at: http://www.businessdictionary.com/definition/evolution.html (Accessed: 15 August 2019).

Carcary, M. et al, 2015. 'Business innovation and differentiation: Maturing the IT capability', IT Professional, Vol. 17, No 2, pp. 46-53.

Curley, M., 2008. The IT Capability Maturity Frameworks. National University of Ireland.

Curley, M. and Kenneally, J., 2011. 'Using the IT Capability Maturity Framework to Improve IT Capability and Value Creation: An Intel IT Case Study', in International Enterprise Distributed Object Computing Conference. Helsinki, pp. 107-115.

Duarte, A. and Da Silva, M. M., 2013. 'Cloud Maturity Model', in 2013 IEEE Sixth International Conference on Cloud Computing. Santa Clara (Califórnia), pp. 606-613.

Flamholtz, E. G. and Aksehirli, Z., 2000. 'Organizational success and failure: An Empirical Test of a Holistic Model', European Management Journal, Vol. 18, No 5, pp. 488-498.

Fong, K., 2010. The Five Elements of Organizational Success. Available at: http://elementalpartners.net/downloads/5_Elements.pdf (Accessed: 20 October 2019). 
Gibson, C. F. and Nolan, R. L., 1974. 'Managing the four stages of EDP growth', Harvard Business Review, Vol. 52(February), pp. 76-88.

Goldbarg, M. and Goldbarg, G., 2012. Grafos: Conceitos, Algoritmos e Aplicações. Rio de Janeiro: Elsevier.

Gross, J. and Yellen, J., 2003. Handbook of Graph Theory. New York: CRC.

Hamel, G. and Prahalad, C. K., 1994. Competing for the future. Boston, Massachussets: Harvard Business School Press. Available at: http://doi.wiley.com/10.1111/j.1467-9310.1996.tb00945.x (Acessed: 20 October 2019).

IVI, 2016. IT Capability Maturity Framework (IT-CMF) - The Body of Knowledge Guide. Maynooth: Innovation Value Institute.

Kaplan, R. S. and Norton, D. P., 1992. The Balanced Scorecard-Measures that Drive Performance, Harvard Business Review. Available at: https://hbr.org/1992/01/the-balanced-scorecard-measuresthat-drive-performance-2 (Accessed: 13 August 2019).

Neely, A., Gregory, M. and Platts, K., 2005. 'Performance measurement system design: A literature review and research agenda', International Journal of Operations \& Production Management, Vol. 25, No 12, pp. 1228-1263.

Nolan, R. L., 1973. 'Managing the computer resource: a stage hypothesis', Communications of the ACM, Vol. 16, No 7, pp. 399-405.

Nolan, R. L., 1979. Managing the crises in data processing, Harvard Business Review. Harvard. Available at: https://hbr.org/1979/03/managing-the-crises-in-data-processing (Accessed: 7 May 2018).

Peppard, J. and Ward, J., 2004. 'Beyond strategic information systems: towards an IS capability', The Journal of Strategic Information Systems, Vol. 13, No 2, pp. 167-194.

Santos, J. R. and Valdesuso, C., 1985. Planejamento Estratégico e Tático da Informática. 2nd edn. Rio de Janeiro: SCI-Editora.

Sink, D. S., 1985. Productivity management: planning, measurement and evaluation, control and improvement. New York: Wiley. Available at: https://trove.nla.gov.au/work/18823864?selectedversion=NBD3748696 (Accessed: 26 June 2018).

\section{APPENDIX A - LIST OF CRITICAL CAPABILITIES}

\begin{tabular}{|l|l|}
\hline Abbreviation & Description \\
\hline AA & Accounting and Allocation \\
\hline BAR & Benefits Assessment and Realization \\
\hline BGM & Budget Management \\
\hline BOP & Budget Oversight and Performance Analysis \\
\hline BP & Business Planning \\
\hline BPM & Business Process Management \\
\hline CAM & Capability Assessment Management \\
\hline CFP & Capacity Forecasting and Planning \\
\hline DSM & Demand and Supply Management \\
\hline EAM & Enterprise Architecture Management \\
\hline EIM & Enterprise Information Management \\
\hline FF & Funding and Financing \\
\hline GIT & Green Information Technology \\
\hline IM & Innovation Management \\
\hline
\end{tabular}


IADIS International Journal on Computer Science and Information Systems

\begin{tabular}{|l|l|}
\hline ISM & Information Security Management \\
\hline ITG & IT Leadership and Governance \\
\hline KAM & Knowledge Asset Management \\
\hline ODP & Organization Design and Planning \\
\hline PAM & People Asset Management \\
\hline PDP & Personal Data Protection \\
\hline PM & Portfolio Management \\
\hline PPM & Programme and Project Management \\
\hline PPP & Portfolio Planning and Prioritization \\
\hline RDE & Research, Development and Engineering \\
\hline REM & Relationship Management \\
\hline RM & Risk Management \\
\hline SAI & Service Analytics and Intelligence \\
\hline SD & Solutions Delivery \\
\hline SP & Strategic Planning \\
\hline SRC & Sourcing \\
\hline SRP & Service Provisioning \\
\hline SUM & Supplier Management \\
\hline TCO & Total Cost of Ownership \\
\hline TIM & Technical Infrastructure Management \\
\hline UED & User Experience Design \\
\hline UTM & User Training Management \\
\hline
\end{tabular}

